\title{
A home-based health information acquisition system
}

\author{
Yan Yan ${ }^{1,2}$, Qi Li ${ }^{1,2}$, Heping $\mathrm{Li}^{3}$, Xuejun Zhang ${ }^{3}$ and Lei Wang ${ }^{1,2 *}$
}

\begin{abstract}
With the development of health consciousness and growing of the aging population, the healthcare demand becomes more and more important. At the same time, recent rapid growth in mobile computing technologies enables telemedicine applications operations on mobile devices. The technologies and demands accelerated different kinds of home-based devices for the aged healthcare and medical treatment. Our focus is on the design of an information system for in-home healthcare, and a new household acquisition terminal device which integrated electrocardiogram (ECG), blood oxygen, blood pressure and body temperature acquirement functions. A set-top box based manipulation method was adopted for operators, the physiological data could be acquired by the corresponding detectors. The parameters were collected and transmitted by set-top box and were received by the data server and sent to the medical staff of a hospital or healthcare center, professional healthcare advice or clinical diagnosis would be provided. Simultaneously, the collected parameters and the feedback clinic advice was stored on the servers which could be accessed by long distance clients such as a mobile smart phone user. The proposed system involved multiple physiologic parameters which led to great potential for universality, and in the terminal side the set-top box and IPTV screen shared scenario brought ease of use. Further progresses were made in the product upgrade because the shared IPTV screen was employed, which might be improved with the family demand. The acquirement instrument got a certification of China State Food and Drug Administration. The entire system was built for commercial achievements and the acquired data had great potential in disease screening and public hygiene research.
\end{abstract}

Keywords: Telemedicine, Home-based healthcare, Information system, Set-top box

\section{Background}

Most developed countries are facing important overall problems with health care services: the increasing elderly and chronic diseases, demand for accessibility and efficiency and so on [1]. These challenges turn home healthcare into one of the fastest growing areas of healthcare provision [2]. Telemedicine methods are widely used in the home healthcare scenarios and applications, which formally defined as the use of medical information exchanged from one site to another via electronic communications to improve a patient's clinical health status, it includes a growing variety of applications and

*Correspondence: wang.lei@siat.ac.cn

1 Shenzhen Institutes of Advanced Technology, Chinese Academic of Sciences, No.1068 Xueyuan Road, Nanshan District, Shenzhen, Guangdong, China

2 Shenzhen Key Laboratory of Low-cost Healthcare, Shenzhen, China

Full list of author information is available at the end of the article services using two-way video, email, smart phones, wireless tools and other forms of telecommunications technology [3]. These technologies permit communications between patient and medical staff with both convenience and fidelity, as well as the transmission of medical, imaging and health informatics data from one site to another. The utilize of telemedicine helps eliminate distance barriers and can improve access to medical or healthcare services, which makes it possible for home-base healthcare and chronic disease monitoring [4-7]. Early forms of telemedicine achieved by telephone is being used in decision-making and follow-up for patients like burning patients [8] and cancer management [9]. As the internet and web technology development, web-based technology used in chronic disease management such as diabetes [10] and HIV/AIDS patients. Mobile and wireless technologies enable the mobile healthcare systems [11,12], not just in the home nursing but also in some mobile scenarios 
like moving vehicles [13]. Telephone or radio has been supplemented with video telephony, advanced diagnostic methods supported by distributed client/server applications, and additionally with telemedical devices to support in-home care [14].

The trend of modern telemedicine or e-Health is multiscenario healthcare application and multi-user data sharing with the development of communication technology and computer science. The utilize of a data sharing mechanism make it possible for remote healthcare and clinical treatment, systems supported multi-user data access which have great importance and potential home-base aged or child care application. The published literature about telemedicine adopted in chronic disease management includes diabetes patients [15], trauma and burns [16], cardiology [17], ischemia stroke [18], dermatology [19] and hypertension [20]. Beyond that, the health monitoring application is becoming more popular especially for the home health care [21-24]. Modern medicine is dependent on the monitoring of patients and early diagnoses and interventions of their conditions and illnesses, which have great significance in elder care and physical examination.

The new medical model which used remote monitoring to get the similar service at home with much lower cost and higher convenience. For the usual health examination, the traditional way could be occurring in a hospital or rehabilitation center, which will not be available in some circumstances such as rural areas and medical condition limited place. Especially for some chronic disease, sustaining hospital-based medical care will bring heavy economic burden for the patients. The home-based healthcare equipments will remarkably reduce cost and inhabited environment. For example, the cardiovascular's diseases (CVD), cardiac patient monitoring systems play a vital role by providing clearly detection and frequent monitoring, to aid the detection and monitoring, telemedicine systems with efficient protocols are developed to help the cardiac patients with the transition from hospital-based care to home-based care, with the improved continuity of care and reduced costs for hospitalization expenses. On the other hand, the world is becoming highly mobile and medical facilities overloaded, in recent years, mobile devices for remotely accessing updated medical information have been adopted in order to reduce errors, improve work flow and enhance data security [25]. The widely used intelligent terminals such as PDA devices bring the probability for mobile diagnosis not to mention the mobile attention. Via the wireless communication networks, gaining the collected data and results analyzed by the remote information analysis center which referred hospital or rehabilitation center where corresponding nurses or doctors can provide professional analysis or diagnosis for the subscribers.
Currently, a lot of home-based healthcare monitoring devices had been developed by different manufacturers in the market. The described wireless in-home physiological monitoring system in [26] was based on multihop relay communications, which adopted a mobile-care device for ECG acquisition, can ubiquitously and continuously monitor the patient's ECG at any time or any place at home without space limit. Sensing chair was developed for a new kind of data acquisition terminal for the telemedicine information system in [27]. RFID technology had been adopted in the home healthcare for the elderly $[28,29]$ detection. The systems in the published literature exist several weak points: first is the acquired physiological parameters are limited which is accepted because some of some systems are designed for one or two special purposes, but still lacking university for home healthcare; second is the data collection devices were weak in the usability especially for the elder in less developed areas who were intensely in complicated operation.

\section{Method \& design}

A home-base healthcare terminal, the remote client service terminal, the data center and the healthcare service provider terminal. The implementation of a home-based data acquisition platform and the client terminal function brought the entire system a data sharing mechanism. In this part, firstly the entire system structure and workflow were described; then the household physiological parameter collection terminal and the house hold subsystem were presented, then the remote clients and data center were explained.

\section{System structure}

As the entire system structure and workflow illustrated in Figure 1, the system working operation process includes three stages, which involves four main constituent parts or subsystems.

In the ontology information acquirement stage, physiological parameters and user information were acquired by the household subsystem, which were collected as the main data source of the system. The private information of the involved user could be set, simultaneously one user item would be created in the data center server, each time the collected physiological parameters would be stored in the chosen user, and that the corresponding user parameter history record was built. Except for the physiological parameters and user information, the video call or voice call were collected to pick up the oral information. A comprehensive information for the health condition or disease state would be collected. Undoubtedly, the information was packaged as data protocol and transmitted to the service center for further use and analysis to build the data sharing mechanism. 


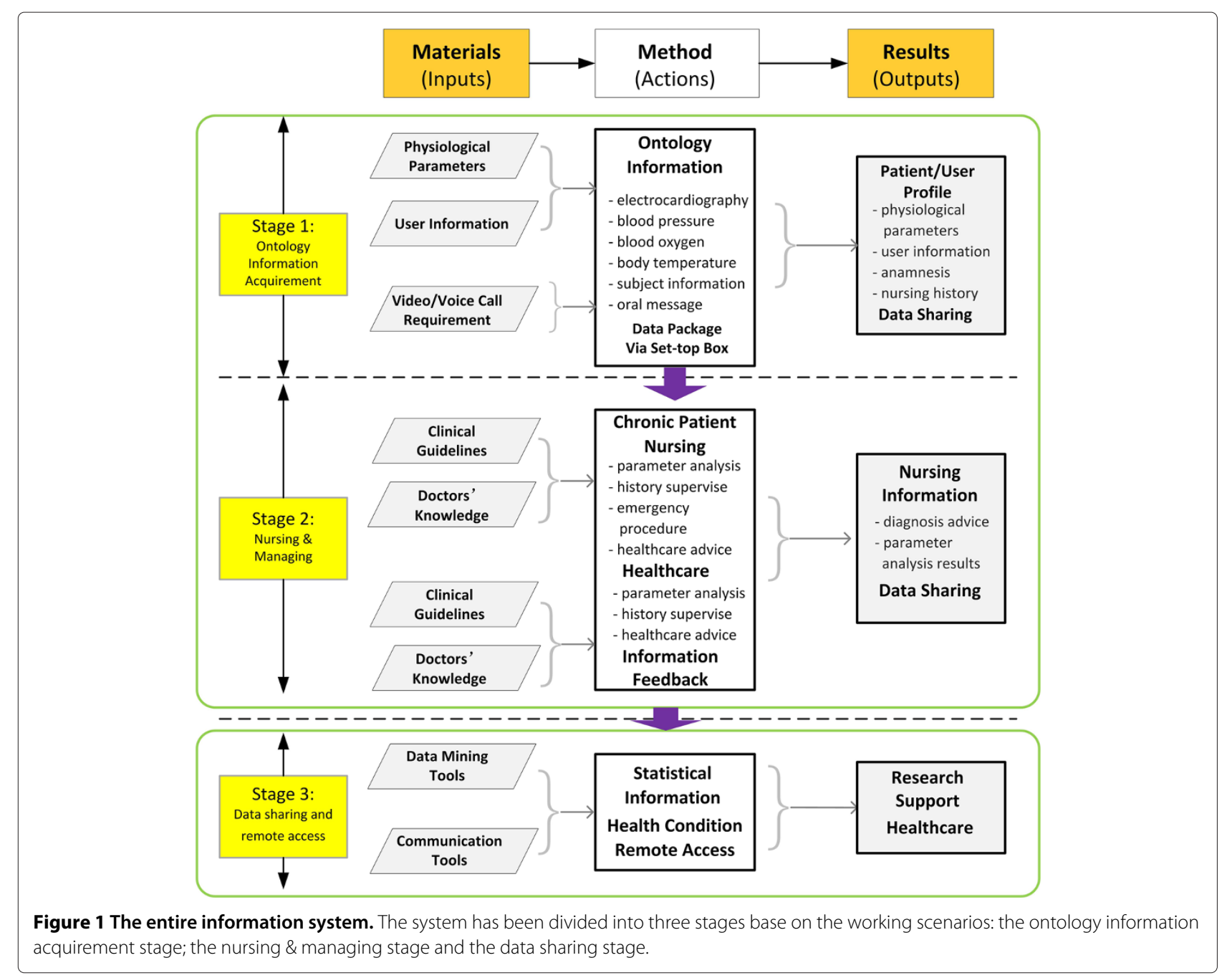

Before the nursing \& managing stage, the acquired patient/user profiles were shared in the service center, the corresponding doctor or nurse has the authority to check the data and profile. A preliminary diagnosis results or healthcare advices would be given by the nursing staffs. Once the homologous diagnosis or judge results provided, the information would be added into the user profile on the server. There is a difference between normal healthcare users and chronic patients, chronic patients would assigned with a more professional doctor because stronger background knowledge is needed.

The third stage is called data sharing and remote access, it includes two parts: one is for the family members of the users; one is for a researcher or decision maker. As for the users' relatives, the access to the related profile and diagnosis or healthcare results would be provided via a given account for the service server. As for the scientist who interested, the data would be provided for the purpose of research, and relevant statistical information would be of research value.

\section{Household acquisition terminal}

Local area network has always been adopted to set up an acquisition system for home health care and it is quite simple and convenient in implementation. However, the expectation of the system is mainly intended in the aged and the high incidence of chronic diseases group. As for the current situation in the developing countries and backward areas, a remote-control unit based operation and an IPTV integrated system might be much more applicable for the elder while the set-top box technology becomes widespread as the digital TV grows more popular. As the house hold acquisition terminal is an important part of the system, in this section, this subsystem would be introduced with three parts: firstly the scenario and work flow of in-home subsystem would be introduced, secondly the hardware configuration of the terminal instrument 
would be presented, lastly the set-box software would be illustrated.

\section{In-home subsystem and scenario}

As Figure 2 shows, the in-home subsystem application scenario includes the user, set-top box, television screen and an acquisition device with its peripheral accessories.

The proposed acquisition terminal acquired the physiological parameters with the peripheral accessories of the instrument. The set-top box and television screen accomplished the user commands reception and data \& information display. The user act as the starter of one event. When users try to use the system to acquire physiological parameters for body examination, the remotecontrol unit or a PDA which supports control applications for the device could be used to choose the appropriate option for acquisition, such as blood pressure or ECG which can be acquired after the corresponding probe or sensors were properly fixed. The terminal device starts booting acquisition process after received the command. The collected data will sent to the MCU for further process after the acquisition procedure finished. Then the processed information will display on the television via a set-top box which gain data from the device via Bluetooth transfer. Depending on the user's choice, the set-top box may transfer the related information to the data center (also called automatic data processing service center) for further doctor diagnosis or analysis. The feedback diagnosis or nursing results will be shown on the television by the set-top box after a certain period.

\section{Hardware configuration}

As in the hardware structure of the in-home terminal instrument illustrated in Figure 2. The instrument system hardware includes front-end circuits, Bluetooth module, storage module, display module and other modules. These modules complete the data acquisition, data communication, data transfer, data storage, information display and other necessary functions such as the button control module and power supply module. For the next important parts would be further described, others would be ignored because they were common sense.

1. Microprogrammed Control Unit:

The MCU worked as the core of the hardware system, in which the data collection, data packaging and data transforming were realized. The popular ARM based MCU was adopted for its low power consumption and ease in development.

2. Data acquisition module: The proposed physiological information includes blood pressure, electrocardiogram, body temperature and blood oxygen, so that this module includes the four function parts and interfaces for the body temperature probe, bloody oxygen probe, blood pressure cuff and electrocardiogram lead system. In the electrocardiogram acquisition system, a seven-lead system were proposed considering the usability and convenience.

3. Data communication module:

The data communication function is an important part of the system. The Bluetooth communication protocols were adopted for the data transfer and

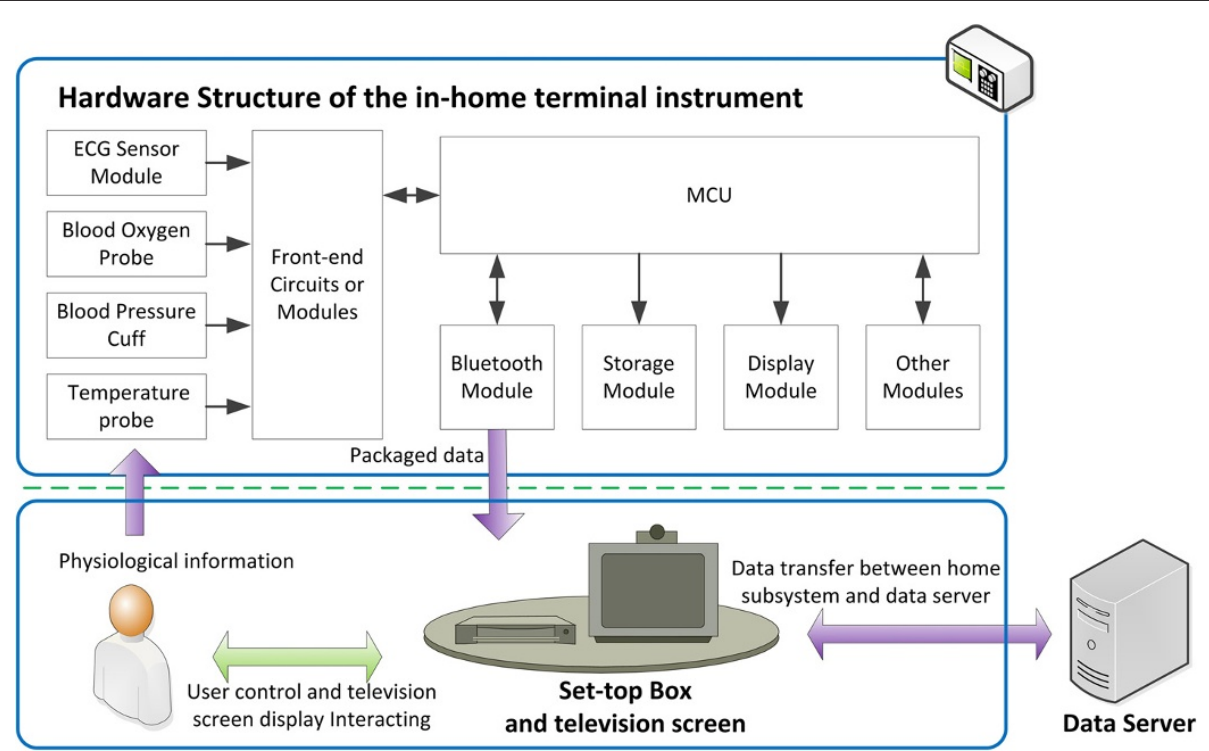

Figure 2 The in-home subsystem. The physiological information were acquired by the terminal instrument and transmitted to the data server for analysis after which the results would be displayed to the users. 
communication, as the scenario is a short-distance and real-time communication application. The Bluetooth connection module was used in data transfer between the set-top box and terminal. A Bluetooth 2.1 protocol was realized in the communication module.

4. Storage Module: As for further development and data backup, the device support storage function. A micro SD card was adopted in the storage module for its convenience and universality.

5. Other modules:

In addition to these main parts, power management module, button control module and OLED display module are designed for the power management, button control and OLED display.

\section{Set-top box software}

A set-top box (STB) or set-top unit (STU) is an information appliance device that generally contains a tuner with connects to a television set and an external source of signal, turning the source signal into content in a form that can then be displayed on the television screen or other display device. The signal source might be an Ethernet cable, a satellite dish, a coaxial cable, a telephone line, broadband over power lines, or even an ordinary VHF or UHF antenna, while the content might include video, audio, Internet web pages, interactive video games, or other possibilities. In the system a Bluetooth communication module, an Ethernet cable, telephone line and cable television were supported for the proposed function and normal TV channel selection, meanwhile the video, audio, Internet web contents were included in the interactive system.

The set-top box is an important part of the household system. As Table 1 illustrated, firstly the set-top box receives user command from the remote control unit, and then send the control order to the device, the device starts the corresponding capture action. Secondly, the set-top box receives processed data via Bluetooth connection, and then try to present the data in an intuitive form for the users. Thirdly, some of the user commands are related to the data upload to the Automatic Data Processing Service Center, so the set-top box also needs complete upload function and further an access to diagnostic information is implemented. The programs are implemented under a Windows XP operating system.

The user interface on the television includes 9 parts as Figure 3 shows, some of the functions were listed in Table 1 for the user operation and set-top box action with its enrolled modules.

1. Information acquisition:

The module includes human-computer interaction for parameter acquirement, which composed the terminal device control command transmitted and received data display, each parameter acquisition mission were designed with special sub UIs and popups.

2. Home healthcare:

The diagnosis or healthcare advice provided in the feedback for the user with acquired physiological parameters.

3. Hospitals service: This module serves as a hospital information for the users, which provide the basic information and the enroll doctors with individual documents and specialties.

4. Video service:

This module supports the direct video calls between users and doctors, the doctors can make a quick diagnosis and detection of the users.

5. Audio service:

The audio interaction between users and doctors was supported.

6. Healthcare document:

This module provides a history data browse.

7. Message region:

The message would be sent by the doctor or other family members.

8. The doctor information display.

9. The user information display.

Table 1 Main functions of the set-top box

\begin{tabular}{|c|c|c|c|}
\hline & User selection & Set-top box software action & Enrolled modules/contents \\
\hline Information acquisition & Parameter pattern selection & Receive the acquired data & Bluetooth with the terminal \\
\hline Information transmission & None & Upload the received data to the serve & Ethernet connection \\
\hline Healthcare service & Diagnosis information display selection & Acquire the feedback & Ethernet \\
\hline Hospital information view & Hospital selection & Acquire the information from the database & Ethernet web content \\
\hline Video communication & Video selection & Build video connection & Ethernet connection \\
\hline Audio communication & Audio selection & Build audio connection & Telephone connection \\
\hline Message communication & Message selection & Text information receipt & Ethernet connection \\
\hline
\end{tabular}

This is where the description of the table should go. 


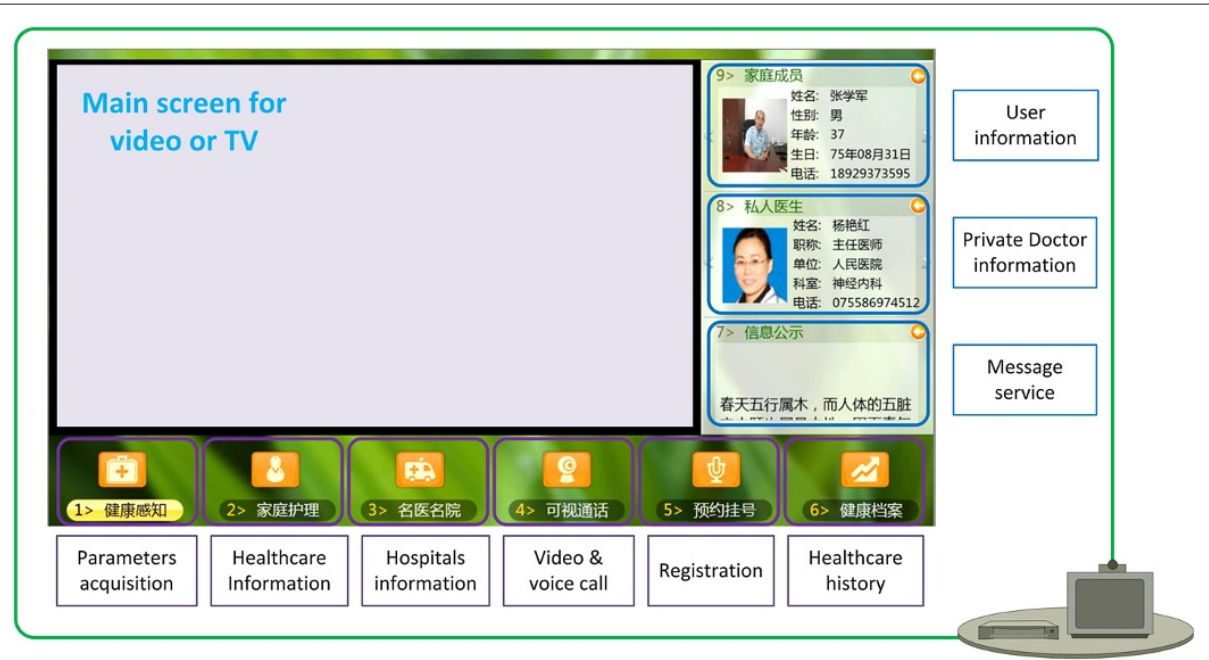

Figure 3 User interface for the set-top box software.

\section{Nursing \& managing}

In the healthcare system, the special medical staff were involved to provide specific medicine knowledge and advise of chronic disease management. A direct connection would be built between the user and medical staff with video call or voice call if the users think necessary, after which a detailed instruction would be made for the users.

The medical staff would be informed about an alert event proactive rather than the staff checking into system and discover themselves, which will be better for the part-time involved doctors or nurses. In the proposed system, once the acquired physiological information updated in the data server, the NM (nursing \& managing) clients would receive alerts from the data server. Then the medical staff check the information and the historical records to judge the health condition of the users. If an emergency abnormality occurred when the medical staff check the physiological parameter, the medical staff would suggest the user going to the hospital or inform the family member and the community nurse for help and the emergency event would be kept as a record. That mechanism is quite important in chronic disease management.

For the medical staff, the operation software was proposed on two different kinds of platform: an Android edition of PDA-based application and a PC based software, which have the exact same functions and mechanisms.

\section{PDA-based mobile clients}

The implementation of the e-health care mobile clients are based on a PDA device with connections to the Internet. In the household scenario, the SET-TOP and television based system acquired the physiological data for the users. And in the doctor user terminal, the related diagnosis results are acquired. This information is stretched in the data center which can be accessed from the Internet. A mobile client device can be used to access the database for the parameters and diagnosis results. This mechanism can make a direct connection between users, doctors with the mobile client so that the mobile client can make a better arrangement for the healthcare object.

The mobile client model contains several Java classes for establishing and closing network connection with the server, downloading information from the server. When a new test and diagnosis mission happens, an alert would be sent and let the mobile user to be able to know the latest condition of the user. The proposed client software is quite simple which received the alter and established connection to the server and get access to the information. The working mechanism is quite similar to the NM client. The feedback could be a message sent back to the server, or quite simply just make a normal telephone call, which would be important especially an emergency happens.

\section{Clients graphical user interface design}

Both in the nursing clients and the normal mobile client (for other family members) side, a smart phone or tablet PC is being used in the usability and generality. The related information can be displayed, scanned, established and analyzed by the users. The view in the mobileclient application could the graphical user interface (GUI) which is composed of several displayable objects include form, alert, graphics, text messages etc. The graphical user interface renders the data into an amicable way. It got the data from the data server and specifies how the model and specified how the data presented to the clients. A view also forwards user credentials and access 
right of mobile client users, a selection of patient to view the information and history information also can be shown.

A friendly GUI is intuitive, clear, consistent and simple in interaction. The user can find all needed functions easily and perform tasks effectively, without getting tangled in secondary issues. In this study, the Android based applications were proposed to the nursing user and normal user, actually they were quite the same in display style and function. They might be considered as one application in two editions, a professional one and an ordinary edition, which would be functionally limited to an ordinary edition. The professional one support a diagnosis results upload and group user management which was for the medical staff and the other is for an ordinary user which only get access to the corresponding records.

\section{The server working frame}

The data server has four major goals:

1. To collect the user test physiological parameters;

2. To collect the diagnosis information (include the communication information);

3. To make alerts and push the needed information;

4. To be the knowledge database of hospital and doctor information and healthcare history information.

Collaborating databases provided data sets and supporting documentation of the recommend hospital and doctor information, as well built connections between the home user and the medical staff. The database consist of a SQLite database that capture the information from the entry the system data. SQLite is a relational database management system contain in a small $C$ programming library, which is not a separate process that is accessed from the client application but an integral part of it [30]. As Figure 4 illustrated, the SQLite database was composed of four parts: the physiological data record , the communication record, the diagnosis record and the medical information (supporting documentation).

The data server service was based on the database. When a new user physiological parameters record enters the server, the data were first added into the corresponding user record, after which an alert was pushed to the related medical staff. A similar process occurred after the medical staff completes the diagnosis action, diagnosis information was added to the database as well. For the communication part, the related information was recorded and added into the database. When a user inquiring behavior happened, the information would be sent to the in-home system from the preset information database. At last, the data server provides a mobile client inquiry mechanism, a remote client can visit the permitted data record, which was significantly important for long distance healthcare for families.

\section{Tests and experiments}

The in-home based healthcare information system developed by our team was in preliminary application. The terminal instruments were manufactured by professional manufacturer, the data server database with a terminal software system were built, the Android application for mobile clients (ordinary users' and professional users' edition) were developed.

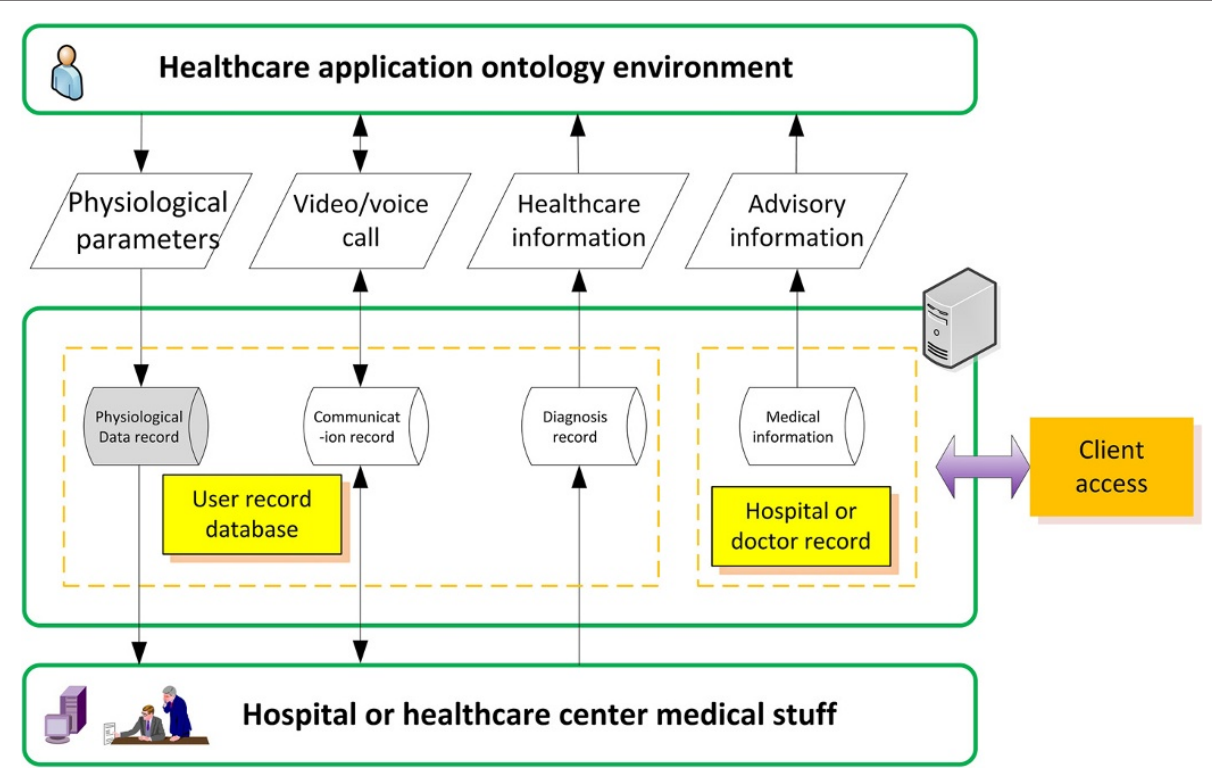

Figure 4 The database structure. The physiological information were acquired by the terminal instrument and transmitted to the data server for analysis after which the results would be displayed to the users. 
Table 2 User acceptance survey

\begin{tabular}{cccccc}
\hline & High & Medium & Acceptable & Low & Very low \\
\hline Number & 0 & 2 & 2 & 10 & 86 \\
\hline
\end{tabular}

The verification for this system includes three parts: the time delay between in-home terminal device and settop box,the in-home terminal response time to the data server; the mobile client response time to the data server. Time delay between the terminal device and set-top box has great impact on the user experience, a shorter delay forms better user experience. Response time is for the remote clients, response time affects the user experience for the medical staff and ordinary users. It had to be mentioned that the data accuracy rate of the terminal device, the terminal device is one kind of armamentarium which should be accredited by official organizations.

\section{Terminal device certifications}

The proposed terminal device was composed of several physiological parameters acquisition functions, as one kind of armamentarium the proposed instrument should be tested by official organizations. The proposed terminal device got a certification from China Food and Drug Administration (used name: State Food and Drug Administration changed since 2013). The electrocardiography evaluation was performed under the $Y Y$ 1079-2008: Electrocardiographic monitors standard, which is based on the ANSI/AAMI EC 13-2002 NEQ. The blood pressure acquisition evaluation was performed under the $Y Y$ 0667-2008: Medical electrical equipment-Part 2-30: Particular requirements for the safety including essential performance of automatic cycling non-invasive blood pressure monitoring equipment, which is based on the IEC 606012-30: 1999 IDT. The electronic thermometer evaluation was performed under the GB/T 21416-2008/XG1-2008: Clinical electronic thermometer, which is based on the EN 1112-00 NEQ. The pulse oximeter evaluation was performed under the YY 0784-2010: Medical electrical equipment-Particular requirements for the basic safety and essential performance of pulse oximeter equipment for medical use, which is based on the ISO 9919:2005.

\section{Bluetooth communication time delay}

Bluetooth is a wireless technology standard for exchanging data over short distances (using short-wavelength radio transmissions in the ISM band from 2400$2480 \mathrm{MHz}$ ) from fixed and mobile devices, creating personal area networks (PANs) with high levels of security [31]. In the proposed system, the Bluetooth worked as a serial port transmission, the Baud rate is $115200 \mathrm{bps}$ which is sufficient in data transmission. Since the set-top box UI software was designed with some popups and interim GUIs, the feel of delay were largely decreased. Here we adopt the subjective method to test the user acceptance of the operation delay, 100 individuals were involved in the acceptance experiment. The survey results are presented in Table 2. We can see that the time delay was acceptable for most users.

\section{Server response time}

To analyze the system performance, we consider the delay and reliability are the most important. Since the information system use TCP/IP protocol in communication among different subsystems, and since TCP employs retransmission of messages in case a message is lost, delay

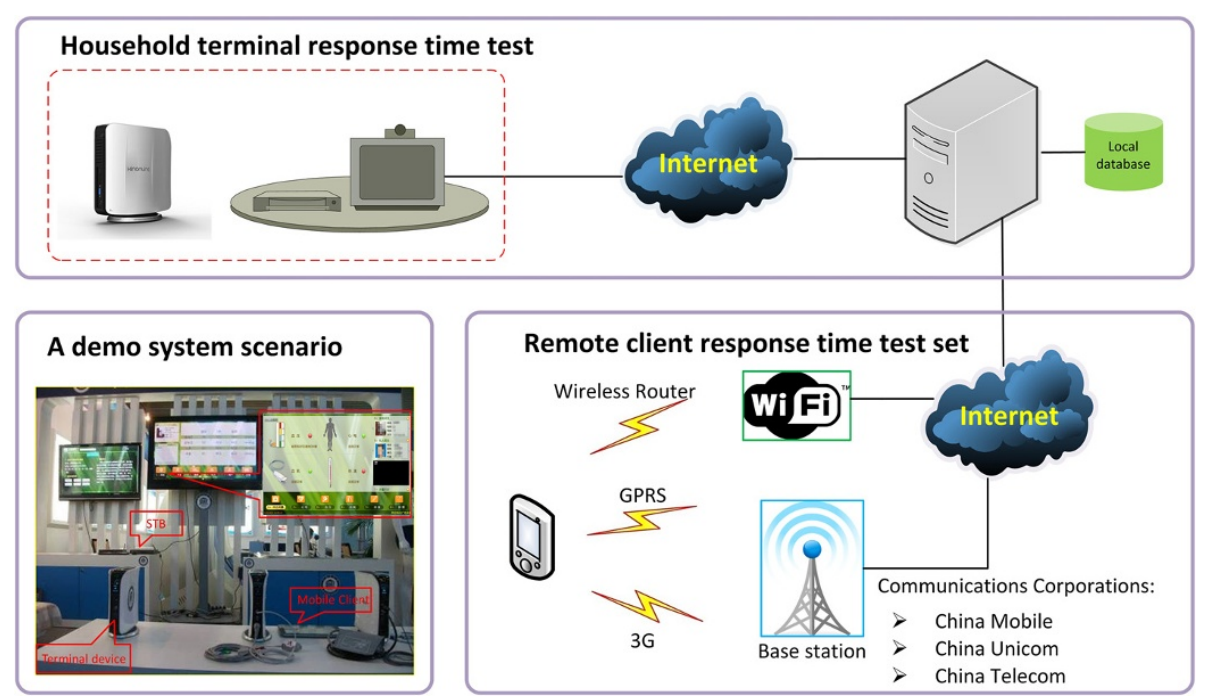

Figure 5 The test settings and scenario. 
Table 3 Household terminal device to server response time

\begin{tabular}{lccc}
\hline Cases sessions & Min $(\mathbf{m s})$ & Max $(\mathbf{m s})$ & Average $(\mathbf{m s})$ \\
\hline Initial \& alert sessions & 76 & 197 & 114 \\
Transmission & 56 & 140 & 95 \\
\hline
\end{tabular}

becomes the most single measurable performance constraint. We use the experiments environment as Figure 5 illustrated, and the method and steps are based on [32]:

- Timestamp S1 at the device is taken when the device sends a message to the server.

- Timestamp S2 at the server is taken when the server receives the message.

- Timestamp S3 at the server is taken when the server sends the response to the device.

- Timestamp S4 at the device is taken when the device receives the response from the server.

The time delay is calculated by:

$$
T_{1}=\frac{(S 4-S 1)-(S 3-S 2)}{2}
$$

The response time test results of the household terminal device to the server are illustrated in Table 3. The same method could be adopted to test the mobile clients, since different network environment has great impacts on the response time and time delay, so we tested the response time in different network environment included the WiFi connection to the Internet with a wireless router, a GPRS connection to the Internet, a $3 G$ connection to the Internet. Typically we chose the GPRS (General Packet Radio Service) network of China Mobile, and the 3G network of China Unicom. Similarly, we tested the initial session and the transmission session in the three kinds of networks, the response time test results were presented in Table 4 and Table 5 below:

\section{Ethical approval}

In this study we analyzed subjective information for 100 observers which was adhered to the tenets of the Declaration of Helsinki.

\section{Discuss \& conclusion}

In this paper, a home-based physiological information acquisition system composed of a in-home system with

Table 4 Mobile client response time - initial session

\begin{tabular}{lccc}
\hline Cases & Min $(\mathbf{m s})$ & Max $(\mathbf{m s})$ & Average $(\mathbf{m s})$ \\
\hline WiFi sessions & 145 & 260 & 178 \\
GPRS sessions & 1239 & 1571 & 1390 \\
$3 G$ & 231 & 368 & 292 \\
\hline
\end{tabular}

Table 5 Mobile client response time - transmission session

\begin{tabular}{lccc}
\hline Cases & Min $(\mathbf{m s})$ & Max $(\mathbf{m s})$ & Average $(\mathbf{m s})$ \\
\hline WiFi sessions & 131 & 226 & 149 \\
GPRS sessions & 1138 & 1462 & 1203 \\
3G sessions & 186 & 294 & 230 \\
\hline
\end{tabular}

a household terminal device, remote clients for medical staff and ordinary user, database and data server software was proposed.

Our system is proposed as a solution for home-based healthcare and chronic disease management. Multiple physiological parameters include blood pressure, blood oxygen, ECG, body temperature and respiration rate were acquired by the household terminal device. Doctor terminals and mobile clients software were accomplished, the former supports the remote monitoring and diagnosis, and the latter can ac-cess parameters and diagnosis information remotely so as to make it possible for long distance caring. Data server was designed as the data accumulator which collects data into a database and a service-provide software for data transfer was implemented.

Evaluation about the information system were proposed, the time delay was surveyed as highly acceptable. For the household device, the response time to the data server was tested and the response time shows good performance. For the remote clients, we tested the server response time under different network environment, the test results showed that a wireless way is slower than an Internet cable connection. However, the response time shows that the results were acceptable when WiFi and $3 G$ Internet connection were used, for a GPRS Internet connection, time delay turned unacceptable in the user experience.

In the proposed system, we adopt the set-top box and television screen as one part of the in-home healthcare subsystem, which is a new idea in the home-based healthcare. The proposed hardware structure is simple and efficient, and no extra display screen was adopted, the healthcare cost is very low. And for the mobile clients, smart phone was able to complete everything, so the cost for the entire information system is low. The operation manner is very convenient, for the elder users, sometime a fashion kind of operation method might not help, but the remote controller for the set-top box with television is widely used in all families, so the users especially for the elderly, they would not be confused in using this machine. The physiological parameters we acquired was comprehensive which would be sufficient for most chronic disease, so the universality of the system is high. The mobile and remote architecture will become a general system model for future E-medicine developments and research. In the elder health care and chronic disease management 
application, the performance of the system is stable and reliable.

\section{Competing interests}

The authors declare that they have no competing interests.

\section{Authors' contributions}

YY was responsible for the system structure design and implementation, data acquisition and analysis. OL participated in data acquisition and data analysis. HL contributed to database implementation. XZ participated in software design and hardware implementation. LW provided the experimental infrastructure and contributed to the result discussion. All authors read and approved the final manuscript.

\section{Acknowledgements}

This research was financed by the National 863 Program of China (Grant No. 2012AA02A604), the National 973 Program of China (Grant No.

2010CB732606), the Guangdong Innovation Research Team Funds for Low-cost Healthcare and Image-Guided Therapy, the Next generation communication technology Major project of National S \& T (Grant No. 2013ZX03005013), the Key Research Program of the Chinese Academy of Sciences and Shenzhen Key Laboratory of Low-cost Healthcare.

\section{Author details}

Shenzhen Institutes of Advanced Technology, Chinese Academic of Sciences, No.1068 Xueyuan Road, Nanshan District, Shenzhen, Guangdong, China. ${ }^{2}$ Shenzhen Key Laboratory of Low-cost Healthcare, Shenzhen, China.

${ }^{3}$ Kinonline Co., LTD, Shenzhen, China.

Received: 8 July 2013 Accepted: 22 October 2013

Published: 1 November 2013

\section{References}

1. Koch S: Home telehealth-current state and future trends. Int J Med Inform 2006, 75:565-576.

2. Boulos MN, Wheeler S, Tavares C, Jones R: How smartphones are changing the face of mobile and participatory healthcare: an overview, with example from eCAALYX. Biomed Eng Online 2011, 10:24.

3. What is telemedicine? http://www.americantelemed.org/learn.

4. Kashem A, Droogan MT, Santamore WP, Wald JW, Bove AA: Managing heart failure care using an internet-based telemedicine system. $J$ Card Fail 2008, 14:121-126.

5. Giordano A, Scalvini S, Zanelli E, Corrà U, Longobardi GL, Ricci VA, Baiardi P, Glisenti F: Multicenter randomised trial on home-based telemanagement to prevent hospital readmission of patients with chronic heart failure. Int $J$ Cardiol 2009, 131:192-199.

6. Della Mea V, Marin D, Rosin C, Zampa A: Web-based specialist support for spinal cord injury person's care: lessons learned. Int J Telemed App/ 2012, 2012:7.

7. Van Allen J, Davis AM, Lassen S: The use of telemedicine in pediatric psychology: research review and current applications. Child Adolesc Psychiatr Clin N Am 2011, 20:55-56.

8. Turk E, Karagulle E, Aydogan C, Oguz H, Tarim A, Karakayali H, Haberal M: Use of telemedicine and telephone consultation in decision-making and follow-up of burn patients: Initial experience from two burn units. Burns 2011, 37:415-419.

9. Ricke J, Bartelink H: Telemedicine and its impact on cancer management. Eur J Cancer 2000, 36:826-833.

10. Azar M, Gabbay R: Web-based management of diabetes through glucose uploads: Has the time come for telemedicine? Diabetes Res Clin Pract 2009, 83:9-17.

11. Jen WY: Mobile healthcare services in school-based health center. Int J Med Inform 2009, 78:425-434.

12. Wu I-L, Li J-Y, Fu C-Y: The adoption of mobile healthcare by hospital's professionals: An integrative perspective. Decis Support Syst 2011, 51:587-596

13. Batistatos MC, Tsoulos GV, Athanasiadou GE: Mobile telemedicine for moving vehicle scenarios: Wireless technology options and challenges. J Netw Comput App/ 2012, 35:1140-1150.
14. Sachpazidis I: Image and medical data communication protocols for telemedicine and teleradiology. PhD thesis. Darmstadt University of Technology; 2008.

15. Gani A, Gribok AV, Lu Y, Ward WK, Vigersky RA, Reifman J: Universal glucose models for predicting subcutaneous glucose concentration in humans. IEEE Trans Inf Technol Biomed 2010, 14:157-165.

16. Wallace DL, Jones SM, Milroy C, Pickford MA: Telemedicine for acute plastic surgical trauma and burns. J Plast Reconstr Aesthet Surg 2008, 61:31-36

17. Bui AL, Fonarow GC: Home monitoring for heart failure management. J Am Coll Cardiol 2012, 59:97-104.

18. Amorim E, Shih MM, Koehler SA, Massaro LL, Zaidi SF, Jumaa MA, Reddy VK, Hammer MD, Jovin TG, Wechsler LR: Impact of telemedicine implementation in thrombolytic use for acute ischemic stroke: the university of Pittsburgh medical center telestroke network experience. J Stroke Cerebrovasc Dis 2013, 22:527-531.

19. Ríos-Yuil JM: Correlation between face-to-face assessment and telemedicine for the diagnosis of skin disease in case conferences. Actas Dermo-Sifiliográficas (English Edition) 2010, 103:138-143.

20. Bobrie G, Postel-Vinay N, Delonca J, Corvol P, SETHI Investigators: Self-measurement and self-titration in hypertension: a pilot telemedicine study. Am J Hypertens 2007, 20:1314-1320

21. Botia JA, Villa A, Palma J: Ambient assisted living system for in-home monitoring of healthy independent elders. Expert Syst App/ 2012, 39:8136-8148.

22. Valls A, Gibert K, Sáncheza D, Batet M: Using ontologies for structuring organizational knowledge in home care assistance. Int J Med Informatics 2010, 79:370-387.

23. Bossen C, Christensen LR, Grönvall E, Vestergaard LS: CareCoor: Augmenting the coordination of cooperative home care work . Int J Med Informatics 2013, 82:189-199.

24. Hung Y-S, Chen K-LB, Yang CT, Deng GF: Web usage mining for analysing elder self-care behavior patterns. Expert Syst App/ 2013, 40:775-783.

25. Goh KW, Lavanya J, Kim Y, Tan EK, Soh CB: A PDA-based ECG beat detector for home cardiac care. In 27th Annual International Conference of Engineering in Medicine and Biology Society. Shanghai: IEEE-EMBS; 2005:375-378

26. Lai C-C, Lee R-G, Hsiao C-C, Liu H-S, Chen C-C: A H-QoS-demand personalized home physiological monitoring system over a wireless multi-hop relay network for mobile home healthcare applications. J Netw Comput Appl 2009, 32:1229-1241

27. Jia G, Zhou J, Yang P, Pan Q, Fu X, Ning G, Cao X, Hu H: A sensing chair design for home based physiological signs monitoring. In 2013 IEEE International Symposium on Medical Measurements and Applications Proceedings (MeMeA). Minneapolis: IEEE; 2013.

28. Hsu H-H, Chen C-C: RFID-based human behavior modeling and anomaly detection for elderly care. Mobile Inf Syst 2010, 6:1229-1241.

29. Raad MW: A ubiquitous mobile telemedicine system for the elderly using RFID. Int J Secur Netw 2010, 5:156-164.

30. Owens M: The Definitive Guide to SQLite. Charlotte: Apress; 2006.

31. David Kammer McNutt G, Senese B, Bray J: Bluetooth Application Developer's Guide: The Short Range Interconnect Solution. New York: Bluetooth Special Interest Group; 2012

32. Kulkarni P, Ozturk Y: mPHASiS: Mobile patient healthcare and sensor information system. J Netwo Comput App/ 2011, 34:402-417.

doi:10.1186/2047-2501-1-12

Cite this article as: Yan et al: A home-based health information acquisition system. Health Information Science \& Systems 2013 1:12. 